\title{
RELIEF OF BRONCHIAL COMPRESSION CAUSED BY A CONGENITAL HEART DEFECT BY REMODELING OF THE AORTIC ARCH
}

\author{
René Prêtre, MD, and Marko I. Turina, MD, Zürich, Switzerland
}

Vascular compression of the tracheobronchial tree can create troublesome respiratory problems after an otherwise successful correction of a cardiac defect. Short arching of the thoracic aorta, by a bowstring effect, or medial position of the descending aorta on the vertebral column can be a cause for compression of the left main bronchus. Anterior traction and fixation of the ascending aorta to the sternum has been the classic approach to tracheobronchial compression, ${ }^{1,2}$ but with inconsistent results. We chose to address the aortic anomaly more directly in 2 patients with compression of the left main bronchus by remodeling an ampler arch of the aorta via a posterior approach.

Patients and surgical technique. A newborn infant with type B interrupted aortic arch and a 6-month-old infant with double-outlet right ventricle underwent remodeling of the aortic arch to relieve compression of the left main bronchus. The second baby, who had a high pulmonary flow and dilated pulmonary arteries, already had preoperative compression of the bronchus. Cardiac physiology and hemodynamic parameters became normal after surgical correction in both infants. Extubation was not possible because of inadequate blood gas exchange and retained tracheobronchial secretions. Examination with a bronchoscope revealed a pulsatile compression of the left main bronchus. Tracheostomy was performed in the first baby. Chest computed tomography revealed a compression of the left main bronchus by a medially positioned proximal descending aorta in the second baby (Fig 1).

By way of a posterior muscle-sparing thoracotomy, the aortic arch and descending aorta were extensively mobilized: the aortic arch up to the anastomotic site on the ascending aorta in the first baby and up to the pericardial aortic reflection in the second; the descending aorta down to the diaphragm in both infants. The first pair of intercostal arteries was ligated and divided; the other pairs were prepared to allow maximal cephalad displacement of the descending aorta. Perioperative bronchoscopic examination showed persistent compression of the bronchus after simple dissection of the aorta and relief

From the Department of Cardiovascular Surgery, University Hospital, Zürich, Switzerland.

Received for publication Aug 24, 1999; accepted for publication Sept 22, 1999.

Address for reprints: René Prêtre, MD, Cardiovascular Surgery, University Hospital, 100 Rämistrasse, CH-8091 Zürich, Switzerland (E-mail: rene.pretre@chi.usz.ch).

J Thorac Cardiovasc Surg 2000;119:173-4

Copyright (C) 2000 by Mosby, Inc.

$0022-5223 / 2000 \$ 12.00+0 \quad \mathbf{1 2 / 5 4 / 1 0 3 3 0 4}$
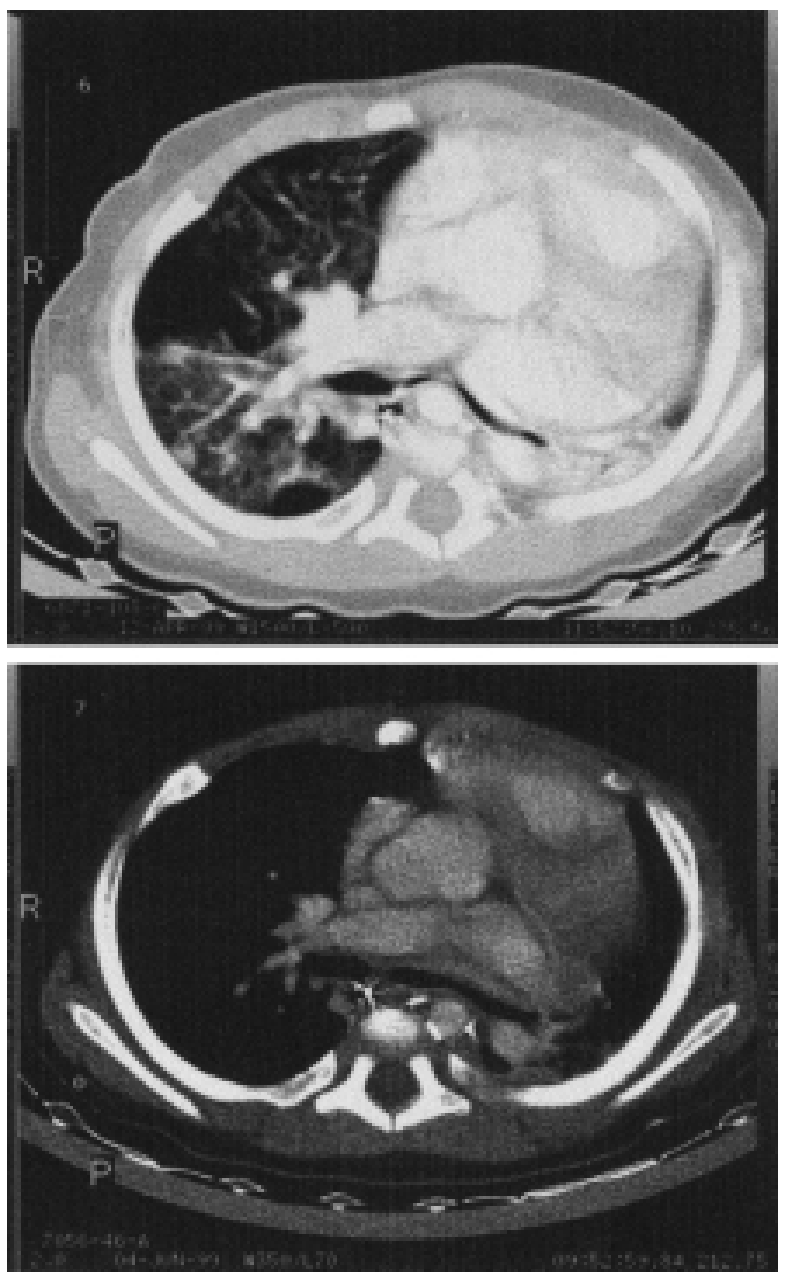

Fig 1. Chest computed tomograms showing compression of the left main bronchus by the proximal descending aorta, which has a medial position above the vertebral column (top). A left shift of the mediastinum has occurred because of preoperative atelectasis of the left lung. After creation of a wider aortic arch, the descending aorta lies more posteriorly, aside from the vertebral column, and has relieved the bronchial compression (bottom).

of the compression after the aorta was pulled away from the bronchus. This was achieved by giving more length to the aortic arch and positioning the proximal descending aorta more posteriorly. The result was a remodeling of the aortic arch to make it wider (Fig 2). The proximal descending aorta 


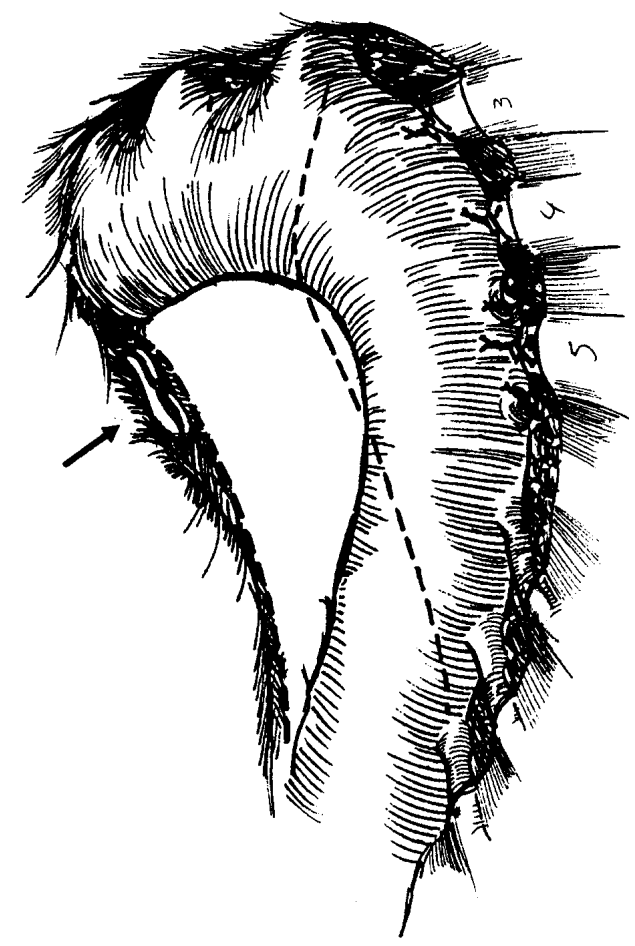

Fig 2. Remodeling of a wider aortic arch after extensive dissection of the descending aorta. The distal aortic arch and proximal descending aorta are fixed to the rib periosteum in a more cephalad and posterior position. Dashed line shows the initial position of the descending aorta with a bowstring effect on the left main bronchus (arrow).

was therefore appropriately fixed with stitches to the periosteum of the underlying ribs.

Results. Vascular compression of the left main bronchus was relieved in both infants. A residual narrowing of $20 \%$ was documented by perioperative and postoperative bronchoscopic studies. Blood gases and respiratory parameters improved and tracheobronchial secretions diminished. The tracheotomy cannula was removed 30 days after the operation in the first baby, and extubation was possible after 14 days in the second baby. Stenting of the left main bronchus, as initially planned, was avoided.

Discussion. Vascular compression of the trachea or a main bronchus occurs with dilatation of the great arteries, congen- ital or surgically created deformities of the aortic arch, or with a combination of these anomalies. ${ }^{1-4}$ A direct approach to dilated arteries, vascular ring, or sling usually results in successful relief of compression. ${ }^{1,2}$ Correction of a cardiac defect to eliminate a volume overload or pulmonary hypertension is often insufficient for relief of severe compression, as exemplified in our second patient. Anterior displacement and fixation of the ascending aorta against the sternum has at times relieved compression on the trachea but inconsistently on the remote left main bronchus. Compression of the left main bronchus often results from a space conflict within the aortic arch. Our approach aimed at remodeling a wider aortic arch appears sound because it directly addresses this space conflict. Normal arching of the aorta is ample and encompasses three quarters of a spire, starting at the aortic anulus and ending at the beginning of the descending aorta. Reduction in the length of the spire, which may occur after operations on the ascending aorta, reduces the radius of curvature and the space within the arch. The risk of a left main bronchus compression is particularly great after correction of an interrupted aortic arch because the gap between the two aortic extremities is wide after resection of ductal tissue, and mobilization of the descending aorta to gain length is limited through an anterior sternotomy.

Widening of the aortic arch relieved compression of the left main bronchus in both of our patients. The gain in length of the aorta obtained after extensive dissection of the descending aorta allowed a more cephalad and posterior coursing of the distal aortic arch and proximal descending aorta, away from the bronchus (Fig 2). It is noteworthy that extensive dissection of the aorta was insufficient to relieve bronchial compression, which was achieved only after widening of the aortic arch.

\section{REFERENCES}

1. McElhinney DB, Reddy VM, Pian MS, Moore P, Hanley FL. Compression of the central airways by a dilated aorta in infants and children with congenital heart disease. Ann Thorac Surg 1999;67:1130-6.

2. Robotin MC, Bruniaux J, Serraf A, et al. Unusual forms of tracheobronchial compression in infants with congenital heart disease. J Thorac Cardiovasc Surg 1996;112:415-23.

3. Worsey J, Pham SM, Newman B, Park SC, del Nido PJ. Left main bronchus compression after arterial switch for transposition. Ann Thorac Surg 1994;57:1320-2.

4. Fadel E, Chapelier AR, Cerrina J, Macchiarini P, Dartevelle PG. Vascular ring causing symptomatic tracheal compression in adulthood. Ann Thorac Surg 1995;60:1411-3. 